\title{
The X-ray emission mechanism of large scale powerful quasar jets: Fermi rules out IC/CMB for 3C 273.
}

\author{
Markos Georganopoulos ${ }^{1,2, a}$ and Eileen T. Meyer ${ }^{3, b}$ \\ ${ }^{1}$ Department of Physics Department, University of Maryland Baltimore County, 1000 Hilltop Circle, Baltimore MD 21250, USA \\ ${ }^{2}$ NASA Goddard Space Flight Center, Code 660, Greenbelt, MD 20771, USA \\ ${ }^{3}$ Space Telescope Science Institute, 3700 San Martin Drive, Baltimore, MD 21218, USA
}

\begin{abstract}
The process responsible for the Chandra-detected X-ray emission from the large-scale jets of powerful quasars is not clear yet. The two main models are inverse Compton scattering off the cosmic microwave background photons (IC/CMB) and synchrotron emission from a population of electrons separate from those producing the radio-IR emission. These two models imply radically different conditions in the large scale jet in terms of jet speed, kinetic power, and maximum energy of the particle acceleration mechanism, with important implications for the impact of the jet on the larger-scale environment. Georganopoulos et al. (2006) proposed a diagnostic based on a fundamental difference between these two models: the production of synchrotron X-rays requires multi-TeV electrons, while the $\mathrm{EC} / \mathrm{CMB}$ model requires a cutoff in the electron energy distribution below $\mathrm{TeV}$ energies. This has significant implications for the $\gamma$-ray emission predicted by these two models. Here we present new Fermi observations that put an upper limit on the gamma-ray flux from the large-scale jet of 3C 273 that clearly violates the flux expected from the IC/CMB X-ray interpretation found by extrapolation of the UV to X-ray spectrum of knot A, thus ruling out the IC/CMB interpretation entirely for this source. Further, the upper limit from Fermi puts a limit on the Doppler beaming factor of at least $\delta<9$, assuming equipartition fields, and possibly as low as $\delta<5$ assuming no major deceleration of the jet from knots A through D1.
\end{abstract}

\section{Introduction}

Large-scale jets with sizes up to $\sim$ Mpc are routinely seen in radio observations of radio-loud AGN, but only more recently has high-resolution imaging with the Hubble Space Telescope (HST) and the Chandra X-ray observatory shown that the knots in many of these large-scale jets often produce significant high-energy radiation. Since the first (serendipitous) Chandra detection of a large-scale $\mathrm{X}$-ray jet in PKS 0637-752 [5], several dozen have been discovered [see 14, for a review], spanning a range from typically lower radio power Fanaroff and Riley (FR) [8] type I to more powerful FR II type radio galaxies.

With high-resolution multi-band imaging, we are now able to build reliable spectral energy distributions (SEDs) for the large-scale jet emission, separate from the blazar core. In many cases, the spectra of the knots appears consistent with a single synchrotron origin from radio to $\mathrm{X}$ rays, as seen in M87 [19], B2 0331+39 [25], and 3C 31 [13], and several others, all notably FR I sources. However, in several of the more powerful (typically FR II) sources, the X-ray spectrum in the knots is clearly much harder and/or higher than would be consistent with the radio-optical synchrotron spectrum, as first observed by [22] and [5] for PKS 0637-752.

\footnotetext{
ae-mail: georgano@umbc.edu

be-mail: meyer@stsci.edu
}

Based on that finding, [23] and [4] suggested that the $\mathrm{X}$-rays could be due to IC/CMB photons by relativistic electrons in the jet (synchrotron self-Compton has been shown to be an inadequate mechanism to produce the observed X-ray flux in these sources, unless the magnetic field in the jet is orders of magnitude below the equipartition value [e.g. 5]). The IC/CMB model has since been applied to other jets with X-rays inconsistent with their radio-optical synchrotron spectra, including the wellstudied source 3C 273 [20], and many more FR II X-ray jets subsequently discovered (e.g., [2, 17, 21]). Generally, the IC/CMB model requires that the jet remain highly relativistic out to the location of the X-ray knots (bulk Lorentz factor $\Gamma \sim 10-20$ ), point close to our line of sight, and have an electron energy distribution (EED) extending down to energies $\sim 10-100 \mathrm{MeV}$, significantly lower than the $\sim 1-10 \mathrm{GeV}$ electron energies traced by $\mathrm{GHz}$ synchrotron radio emission. To produce the observed X-ray flux, however, in view of the low radiative efficiency of these electrons, IC/CMB requires high, sometimes superEddington jet kinetic power [7, 24]. Also, the small angle to the line of sight in several cases requires $\sim$ Mpc-scale de-projected jet lengths, as long as the longest radio galaxies observed [7].

Deep HST imaging photometry of the knots in PKS 1136-135 (previously modeled by [21] as an IC/CMB source) reveals similar 'improbability' issues with the 
IC/CMB model, with observed optical polarization exceeding 30\%; applying the IC/CMB model requires a significantly super-Eddington jet longer than a Mpc, forming $\mathrm{a} \sim 2.5^{\circ}$ angle to the line of sight and having a Doppler beaming factor $\delta>20$ [2].

An alternative explanation for the X-rays in powerful sources is synchrotron emission from an additional electron energy distribution (EED) $[11,12,24]$. Because the synchrotron emission mechanism is far more efficient than IC/CMB, it does not require the high Lorentz factors, extreme jet lengths or near-Eddington jet powers, as the IC/CMB model does in several cases [7, 15, 24]. However, it is not clear what physical mechanism might produce this second EED, and in many cases the observed SED [e.g. 17] requires the high-energy particle population to have a difficult-to-explain low-energy cutoff at $\sim \mathrm{TeV}$ energies, where fast cooling is unavoidable.

One of the best-studied large-scale jets is seen in the powerful nearby $(\mathrm{z}=0.158)$ quasar $3 \mathrm{C} 273$. Imaging in all bands reveals similar features, with a knotty jet beginning about 12" from the blazar core and extending a further 12" downstream. Extensive observations with HST, Spitzer, and Chandra have revealed that the SEDs of all knots in the jet are characterized by two components, a low-energy one having a cutoff above $\approx 5 \times 10^{13} \mathrm{~Hz}$ and a high-energy one connecting the optical-UV and X-ray data $[10,11,24]$. Ir was shown [9] that while the radio to $\mathrm{X}$ ray SED of this source alone cannot discriminate between the IC/CMB and synchrotron models, $\gamma$-ray observations, specifically with Fermi, may be able to do so. If the X-rays from the $3 \mathrm{C} 273$ jet are due to IC/CMB, a hard, steady spectrum is also expected in the gamma-rays by extension [9]. If Fermi detects this emission or puts limits on it below what is expected by extrapolation from the X-rays, the IC/CMB model will be ruled out.

The competing IC/CMB and synchrotron models imply radically different views of the large-scale jet power, bulk Lorentz factor, and the efficiency of particle acceleration, and thus results to very different impacts on the host galaxy and surrounding environment. The persistently open question of the nature of the X-rays is critical not only for understanding jet physics but also for our understanding of AGN activity as a feedback mechanism in galaxy formation, yet until now no conclusive evidence has arisen to eliminate either model.

Here, we revisit the predictions of [9] and analyze the $\gamma$-ray output of 3C 273 for evidence of the expected hard, flat spectrum from IC/CMB which has been suggested as the source of the X-rays in this and other powerful largescale jets. In Section 2, we discuss the method of the Fermi data analysis and our finding that no IC/CMB emission has been detected. In Section 3 we discuss the resulting upper limit on the IC/CMB emission along with an analysis of the entire SED of the 3C 273 large-scale jet. In Section 4 we derive a limit for the Doppler factor based on our Fermi result.

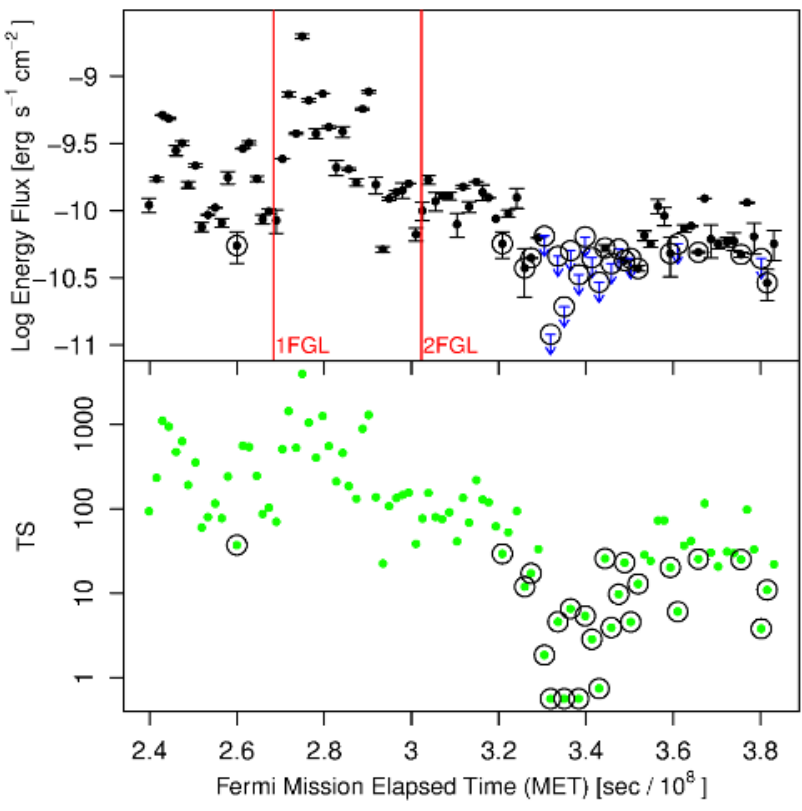

Figure 1. Upper Panel: Nearly 4.5 year lightcurve of 3C 273 (2008 Aug 4 to 2013 March 11) in bins of equal GTI time (7.5 days), with total Fermi band ( $100 \mathrm{MeV}$ to $100 \mathrm{GeV}$ ) energy flux versus Mission Elapsed Time (MET). The ends of the time range for the 1FGL and 2FGL catalogs are noted with red lines. Detections are shown as points with error bars, while upper limits (when the TS of the source was $<10$ ) are shown as arrows. The circled points in both panels are the 25 lowest bins which were combined for the final analysis. Lower Panel: The TS of the source versus Fermi MET. The significance of an individual bin is roughly $\sqrt{T S}$.

\section{Fermi Analysis of 3C 273}

The gamma-ray analysis is complicated by the fact that Fermi lacks the spatial resolution to resolve the blazar core separately from the large scale jet. The knots of the jet of 3C 273 range from $~ 12-24$ " from the core, while the Fermi angular resolution ranges from $3.5^{\circ}$ at $100 \mathrm{MeV}$ down to $\sim 0.15^{\circ}$ above $10 \mathrm{GeV}$, at which point it is still above an order of magnitude larger than the distance of the large-scale jet. Further, the blazar appears to dominate the total emission from 3 C 273 , as seen in the extreme variability of the total Fermi-band lightcurve (Figure 1).

The lightcurve shown was initially computed using bins of equal Good Time Interval (GTI) time, totalling 648000 seconds (7.5 days) per bin, corresponding to a range of 15-23 days in real time. For each time bin, the flux (or upper limit) of 3C 273 was found using the standard pipeline tools (version v9r27p1), and the latest instrument response function (P7SOURCE_V6). Using a region of interest (ROI) of 7 degrees, all sources listed in the twoyear catalog [2FGL; 18] within 15 degrees of the position of 3C 273 were included in the initial model in the unbinned likelihood analysis (in some bins, known sources which were undetected were removed in order to gain convergence of the likelihood model). In all time periods, 3C 273 was modeled as a simple powerlaw, with spectral in- 
dex and normalization free. The total time range analyzed corresponds to Fermi Mission Elapsed Time (MET) 2

The results of the lightcurve analysis are shown in Figure 1 , as the total flux from $100 \mathrm{MeV}$ to $100 \mathrm{GeV}$ versus the central MET of the corresponding bin in the upper panel, and test statistic (TS, roughly equivalent to significance squared) versus the latter in the lower panel. Previous calculations (G06) have shown that it may be possible to detect the expected hard, steady component from an IC/CMB component when the competing blazar emission is at a minimum. As can be seen from Figure 1, the blazar varies nearly 2 orders of magnitude in flux over the period studied. While an overall decrease in flux can be seen over the four years, it is clear that the core exhibits significant short-term variability with timescales on the order of the bin widths.

In order to gain the increased sensitivity of a longer integration time on the source while avoiding times where the blazar may come 'up' during an otherwise quiescent period, we used a progressive binning approach, in which the lightcurve bins were ordered according to the total 100 $\mathrm{MeV}-100 \mathrm{GeV}$ flux. Beginning with the lowest flux time period, we then added the next-highest bin (not necessarily contiquous) in succession and re-ran the likelihood analysis for the combined timeframe. At each step, the SED was divided into the five 'standard' energy ranges used in the 2FGL: $100-300 \mathrm{MeV}, 300 \mathrm{MeV}-1 \mathrm{GeV}, 1-3 \mathrm{GeV}$, 3-10 GeV, and 10-100 GeV. When the TS of a given energy bin was less than 10, an upper limit was calculated. Overall, the flux calculations behaved as expected: initially all bands were upper limits, which became progressively lower as more time bins were used, up to the point where the blazar was detected, when the flux values began increasing in the lower-energy bins.

The two highest energy bins (3-10 GeV and 10-100 $\mathrm{GeV}$ ) gave the lowest upper limit fluxes during the entire analysis, starting from $1.9 \times 10^{-11}$ and $3.6 \times 10^{-11} \mathrm{erg} \mathrm{s}^{-1}$ $\mathrm{cm}^{-2}$ for the single lowest-flux bin, down to the lowest upper limit values of $4.9 \times 10^{-13}$ and $2.5 \times 10^{-12} \mathrm{erg} \mathrm{s}^{-1} \mathrm{~cm}^{-2}$, respectively, after the 25 lowest bins were analyzed together. The inclusion of bins after the 25 th lowest only increased fluxes (or upper limits) in all energy bands, so we take these latter values as the lowest possible limits for the 3-10 GeV and $10-100 \mathrm{GeV}$ energy ranges, at the $95 \%$ level $(2 \sigma)$. We note that alternative methods of ordering the bins are possible (such as strictly on upper limit flux value, or by TS); these methods give practically identical results (nearly the same ordering and a minimum flux in the final two bins within a few percent of the above values). The final 5-band SED points (including the first three energy bins where the blazar emission is detected) are shown in Figures 2 and 3. It is clear that the first three bins are a representation of the low-level blazar SED, which is apparently peaking before the Fermi band and rapidly falling off in the high-energy range. The two upper limits shown are thus upper limits for both the blazar emission and the expected hard, steady component from IC/CMB, with the 3-10 GeV limit being the most constraining.

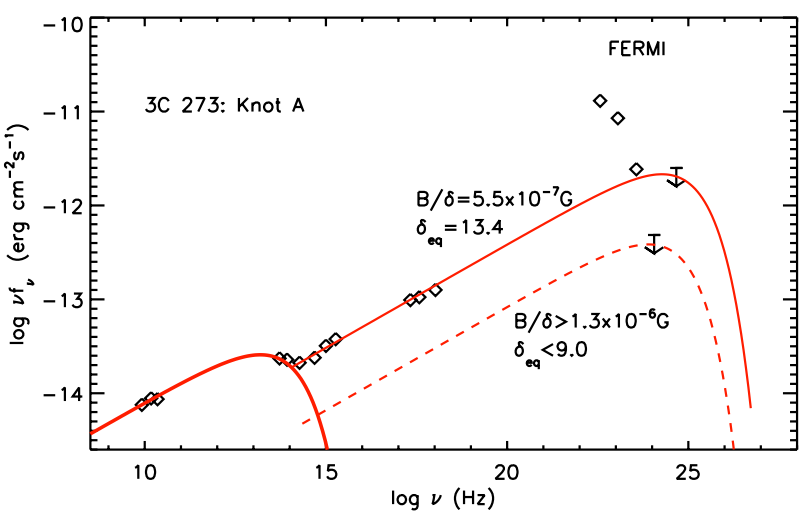

Figure 2. The SED of knot A (data from [24] and [10, 11]), along with the Fermi measurements and upper limits described in $\S 2$. The thick solid line is the parametric fit of the synchrotron SED. The thin solid line is the IC/CMB emission required to fit the UV - X-ray emission of knot A. Note that this unavoidably overproduces the Femi upper limit in the $3-10 \mathrm{GeV}$ band, ruling out the IC/CMB model for the X-ray emission of knot A. The broken line is the highest level the IC/CMB component can have without violating the $3-10 \mathrm{GeV}$ band Fermi $2 \sigma$ upper limit flux of $4.9 \times 10^{-13} \mathrm{erg} \mathrm{s}^{-1} \mathrm{~cm}^{-2}$.

\section{IC/CMB for knot $A$ is ruled out}

In the context of the IC/CMB model, the level of the anticipated $\mathrm{GeV}$ emission is predetermined by the requirement that IC/CMB emission gives the observed X-ray flux. In other words, for a given radio to optical synchrotron SED there is an one-to-one correspondence between the $\mathrm{X}$-ray and the GeV spectrum (see [9] and discussion following). If the anticipated large scale jet $\mathrm{GeV}$ emission from IC/CMB is higher than the low state GeV flux of $3 \mathrm{C}$ 273 , the IC/CMB model is simply ruled out as the source of the X-rays.

Consider the synchrotron SED of knot A, as shown in Figure 2 and modeled phenomenologically as a power-law with an exponential cutoff, following [24]. The relativistic electrons that produce the synchrotron emission will (unavoidably) also inverse Compton scatter the CMB photons, producing an $\mathrm{IC} / \mathrm{CMB}$ component, with a level depending on the characteristics of the jet. As discussed in G06, the IC/CMB SED will be identical to the synchrotron one with a shift in peak frequency

$$
\frac{v_{c}}{v_{s}}=\frac{2 \pi m_{e} c(1+z) v_{0}}{e(B / \delta)}=6.6 \times 10^{4} \frac{\delta}{B / B_{0}}=6.6 \times 10^{8} \delta^{2},
$$

and a shift in peak luminosity

$$
\frac{L_{c}}{L_{s}}=\frac{32 \pi U_{0}(1+z)^{4}}{3(B / \delta)^{2}}=2.5 \times 10^{-11}\left(\frac{\delta}{B / B_{0}}\right)^{2}=2.5 \times 10^{-3} \delta^{4}
$$

where $v_{c}$ and $v_{s}$ are the peak EC and synchrotron frequencies, $L_{c}$ and $L_{s}$ are the peak EC and synchrotron luminosities, $e$ and $m_{e}$ are the electron charge and mass, $\mathrm{B}$ is the comoving magnetic field and $B_{0}=1 \mathrm{G}, v_{0}=1.6 \times 10^{11} \mathrm{~Hz}$ is the CMB peak frequency at $z=0, U_{0}=4.2 \times 10^{-13} \mathrm{erg}$ $\mathrm{cm}^{-3}$ is the CMB energy density at $z=0, \delta$ is the Doppler 


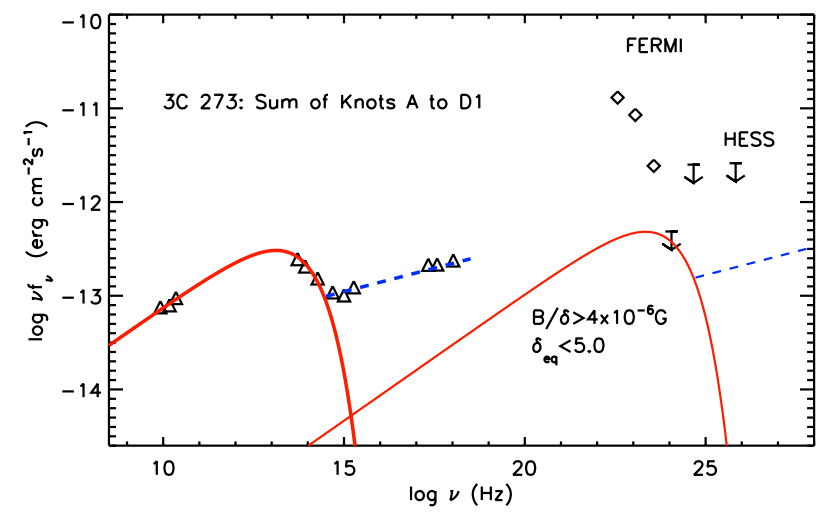

Figure 3. The SED of the jet from knot A to knot D1 (data from [24] and [10]), along with the Fermi measurements and upper limits described in $\$ 2$, and with a HESS upper limit [1]. The thick solid line is the parametric fit of the synchrotron SED. The thick broken straight line is the SED of the UV - X-ray component, modeled as a power-law. Having excluded IC/CMB as the X-ray pemission mechanism, we assume that this is of synchrotron nature. The thin solid line is the maximum amplitude the IC/CMB SED produced by the same electrons producing the synchrotron thick solid line SED can have without violating the 3-10 GeV band Fermi upper limit. The thin broken line is the the IC/CMB SED that results from the same electrons that produce the UV-X-ray synchrotron emission.

factor of the jet, and the last part of each equation holds for equipartition conditions $\left[B \delta \approx 10^{-4} \mathrm{G} ; 10\right]$.

As $B / \delta$ decreases (or as $\delta$ increases if we assume an equipartition field), the IC/CMB SED moves to higher $v_{c}$ and $L_{c}$. To reproduce the UV - X-ray observations of knot A, we require a $B / \delta=5.5 \times 10^{-7} \mathrm{G}\left(\delta_{e q}=13.4\right.$ assuming equipartition). This determines, without any freedom, the anticipated $\mathrm{GeV}$ emission. As can be seen in Figure 2, the level of the IC/CMB emission at $\mathrm{GeV}$ energies violates the upper limit of the 3-10 GeV band, ruling out the IC/CMB interpretation for the knot A X-ray emission of 3C 273.

\subsection{Constraints from the A to D1 knot jet}

So far we have only used knot A, which turns out to be the only knot whose $\mathrm{GeV}$ anticipated emission violates the Fermi 1.0-3.0 GeV band limit. To proceed further we need to consider collectively the emission from the jet. This requires the assumption that the jet flow is characterized by a single Lorentz factor and magnetic field. Radio polarization observations [6] show that the magnetic field polarization direction of the jet runs roughly parallel to the jet from knot A all the way to knot D1. Beyond knot D1 the magnetic field polarization turns abruptly to become orthogonal to the jet axis, as one would expect from a shock that decelerates the flow, compresses the plasma, and amplifies the component of the magnetic field orthogonal to the jet axis. The polarization is suggestive of a jet that does not decelerate substantially from knot A to knot D1, but decelerates efficiently past knot D1. It is, therefore, plausible that the flow from knot A to D1 is characterized by a single Doppler factor. We can also approximately

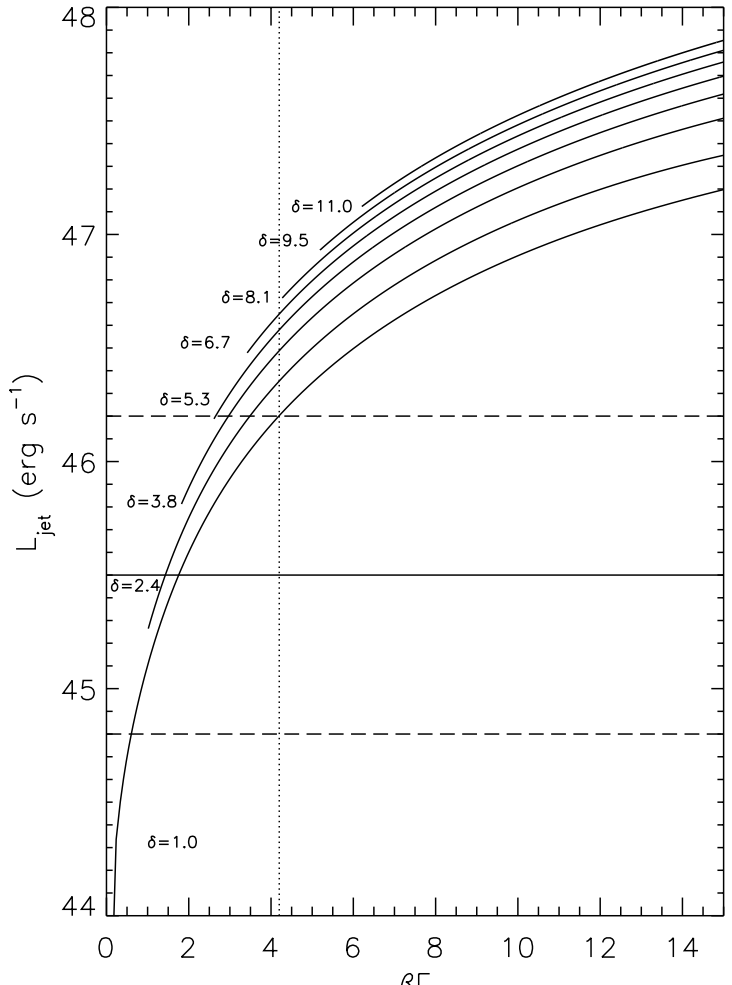

Figure 4. Minimum jet power $L_{j e t}$ as a function of $\beta \Gamma$ for a range of $\delta$. The solid and broken horizontal lines represent the jet power estimate of $L_{j e t}=10^{45.5 \pm 0.7} \mathrm{erg} \mathrm{s}^{-1}$ from the X-ray cavity scaling. Jet configurations with $L_{j e t}>10^{46.2} \mathrm{erg} \mathrm{s}^{-1}$ are disfavored, leading to an upper limit of $\Gamma \gtrsim 4.2$ for the jet.

assume that the magnetic field does not vary significantly from knot A to knot D1, driven by the fact that the equipartition magnetic field of all knots is the same within less than a factor of 2 [10].

Based on the assumption that a single Doppler factor and magnetic field describe the jet from knot A to knot D1 we can further constraint the description of the jet. In Figure 3 we plot the SED of the total flux from knot A up to knot D1, along with our Fermi constraints and previously existing constraints from HESS observations [1]. As can be seen, to satisfy the $3-10 \mathrm{GeV}$ band Fermi limit we require $B / \delta>4.0 \times 10^{-6} \mathrm{G}$, or, assuming equipartition, $\delta_{e q}<5$. The existing shallow TeV limits [3.9 h of HESS observations, no de-absorption applied; 1] do not provide useful constraints, but future $\mathrm{TeV}$ observations with the planned Cherenkov TeV Array (CTA) may be able to detect this component.

\section{An upper limit on the jet bulk Lorentz factor}

We present here a model-dependent upper limit on $\Gamma$, based on an estimate of the jet power scaled from the lowfrequency radio flux of $3 \mathrm{C} 273$, made possible by the the scaling relation between kinetic jet powers estimated by the X-ray cavity method and the low frequency radio lobe 
emission [3]. According to this scaling, the jet power of 3C 273 is $L_{\text {jet }}=10^{45.5 \pm 0.7} \mathrm{erg} \mathrm{s}^{-1}$ [3]. Jet configurations that do not agree with this jet power range are disfavored. Additionally, we make use of the SED of knot A and note that, assuming that the entire radio to X-ray emission comes from the same region, the only frequencies where an electron cooling break can be manifested is either at $10^{13.5} \mathrm{~Hz}$ or above $10^{18} \mathrm{~Hz}$, given that no break is observed between the UV and X-ray observations. In the first case, the optical to X-ray emitting electrons are cooled, requiring a hard electron injection with $n_{i n j}(\gamma) \propto \gamma^{-1.5}$, for what we assume is a second synchrotron component. In the second case the optical to X-ray emitting electrons escape the emission region before cooling and electron injection is steeper by one, with $n_{i n j}(\gamma) \propto \gamma^{-2.5}$. This second case of no cooling up to $10^{18} \mathrm{~Hz}$ requires that a region smaller than the optical jet lateral size $(\sim 1 \mathrm{kpc})$ is responsible for the UV to the X-ray emission. Returning to the first case, the curves plotted in Figure 4 are curves of constant $\delta$. For a given $\Gamma$ the magnetic field that results to a cooling break at $10^{13.5} \mathrm{~Hz}$ is calculated, and from this the electron power needed to produce the SED. By adding to that the Poynting flux, a total jet power is calculated that is a lower limit to the actual jet power, because it does not include protons or thermal electrons. The configuration is disfavored if this jet power is higher than $10^{46.2} \mathrm{erg} \mathrm{s}^{-1}$, the upper limit of expected jet power from the $\mathrm{X}$-ray cavity scaling. As can be seen in Figure 4, this results to $\Gamma \approx \beta \Gamma \lesssim 4.2$.

Using upper limits to the Fermi flux of the LSJ of $3 \mathrm{C}$ 273, we rule out IC/CMB being the X-ray emission mechanism of knot A. This result does not depend on any assumptions of equipartition or jet content and is, therefore, robust. Assuming equipartition, we set an upper limit to the jet Doppler factor, $\delta \leq 5$. Finally, adopting an upper limit to the jet power, derived from the X-ray cavity scaling [3], we find $\Gamma \lesssim 4.2$.

Our result leaves as the only alternative a synchrotron nature for the X-ray emission. This means that in situ particle acceleration takes place that accelerates electrons at least up to $\sim 30-100 \mathrm{TeV}$. It is not clear what particle acceleration mechanism produces this second EED. If we assume that this population cools before it escapes the emission region, a very hard electron injection is required $\left(n_{i n j}(\gamma) \propto \gamma^{-1.5}\right)$. On the other hand, if the electrons escape the emission region uncooled, a steeper electron injection is required $\left(n_{i n j}(\gamma) \propto \gamma^{-2.5}\right)$, but this requires that the emission region is significantly smaller than $1 \mathrm{kpc}$. For $\delta=\Gamma=5$ the maximum size of this emitting region is $\sim 100 \mathrm{pc}$, corresponding to a variability timescale of $\sim 70$ years [note that X-ray variability with a timescale of a few years has been observed for a kpc scale knot in the LSJ of Pictor A; 16]. We finally note that while IC/CMB is ruled out in $3 \mathrm{C} 273$, it is possible that other powerful LSJs produce X-rays through IC/CMB. In future work, we plan to use Fermi to identify the X-ray emission mechanism for additional powerful X-ray detected LSJs.
MG acknowledges support from Fermi grant NNX12AF01G. EM acknowledges support from Fermi grant NNX10AO42G.

\section{References}

[1] Aharonian, F., Akhperjanian, A. G., Bazer-Bachi, A. R., et al. 2005, A\&A, 441, 465

[2] Cara, M., Perlman, E., Uchiyama, Y., et al. 2013, ApJ, 773, 186

[3] Cavagnolo, K. W., McNamara, B. R., Nulsen, P. E. J., et al. 2010, ApJ, 720, 1066

[4] Celotti, A., Ghisellini, G., \& Chiaberge, M. 2001, MNRAS, 321, L1

[5] Chartas, G., Worrall, D. M., Birkinshaw, M., et al. 2000, ApJ, 542, 655

[6] Conway, R. G., Garrington, S. T., Perley, R. A., \& Biretta, J. A. 1993, A\&A, 267, 347

[7] Dermer, C. D., \& Atoyan, A. 2004, ApJ, 611, L9

[8] Fanaroff, B. L., \& Riley, J. M. 1974, MNRAS, 167, $31 \mathrm{P}$

[9] Georganopoulos, M., Perlman, E. S., Kazanas, D., \& McEnery, J. 2006, ApJ, 653, L5

[10] Jester, S., Röser, H.-J., Meisenheimer, K., \& Perley, R. 2005, A\&A, 431, 477

[11] Jester, S., Harris, D. E., Marshall, H. L., \& Meisenheimer, K. 2006, ApJ, 648, 900

[12] Hardcastle, M. J. 2006, MNRAS, 366, 1465

[13] Hardcastle, M. J., Worrall, D. M., Birkinshaw, M., Laing, R. A., \& Bridle, A. H. 2002, MNRAS, 334, 182

[14] Harris, D. E., \& Krawczynski, H. 2006, ARA\&A, 44, 463

[15] Jorstad, S. G., \& Marscher, A. P. 2004, ApJ, 614, 615

[16] Marshall, H. L., Hardcastle, M. J., Birkinshaw, M., et al. 2010, ApJ, 714, L213

[17] Mehta, K. T., Georganopoulos, M., Perlman, E. S., Padgett, C. A., \& Chartas, G. 2009, ApJ, 690, 1706

[18] Nolan, P. L., Abdo, A. A., Ackermann, M., et al. 2012, ApJS, 199, 31

[19] Perlman, E. S., \& Wilson, A. S. 2005, ApJ, 627, 140

[20] Sambruna, R. M., Urry, C. M., Tavecchio, F., et al. 2001, ApJ, 549, L161

[21] Sambruna, R. M., Gambill, J. K., Maraschi, L., et al. 2004, ApJ, 608, 698

[22] Schwartz, D. A., Marshall, H. L., Lovell, J. E. J., et al. 2000, ApJ, 540, L69

[23] Tavecchio, F., Maraschi, L., Sambruna, R. M., \& Urry, C. M. 2000, ApJ, 544, L23

[24] Uchiyama, Y., Urry, C. M., Cheung, C. C., et al. 2006, ApJ, 648, 910

[25] Worrall, D. M., Birkinshaw, M., \& Hardcastle, M. J. 2001, MNRAS, 326, L7 